\title{
A Novel Efficient Stopping Criterion for BICM-ID System
}

\author{
Xiao Ying, Li Jianping \\ Communication University of China
}

\begin{abstract}
This paper devises a novel efficient stopping criterion for bit-interleaved coded modulation with iterative decoding (BICM-ID) system. The new criterion is based on the absolute value of the difference of log-likelihood-ratio (LLR) that between the outputs of the $(i)_{\text {th }}$ iteration and the $(i-1)_{\text {th }}$ of the soft-input soft-output (SISO) decoder. Simulation results comparing the proposed scheme with the widely used cross-entropy (CE) stopping criterion show that although a little more iteration, the proposed scheme can get excellent performance as $C E$ in terms of bit-error-rate (BER) and can get coding gains at high signal-to-noise ratios (SNRs). More importantly, the proposed new criterion doesn't need any exponential computation, which has greatly reduced the complex computation in the BICM-ID receiver. In addition, detailed discussions regarding various parameters that may affect the decoding performances are also provided.
\end{abstract}

\section{Introduction}

Bit-interleaved coded modulation with iterative decoding (BICM-ID) is a smart scheme for its remarkable performances both in AWGN and in Rayleigh fading channels [1]. The iterative decoding algorithm at the receiver especially makes the BICM-ID a very promising technique for the capability of achieving large coding gains without bandwidth expansion $[2,3]$. The iterative decoding process is between the soft-input soft-output (SISO) decoder and the demapper, which is different from turbo iteration. In turbo receiver, the iterative process is between its two inner SISO decoders. The similarity of the two iterative decoding algorithms is that they are both designed to achieve the global optimum through a step-by-step local search. In the iteration cycle, the bit-error-rate (BER) decreases as the iteration number increases, but the incremental improvement gradually diminishes. The conventional iterative decoding algorithm is a scheme with fixed iteration number $M$. Usually $M$ is set with the worst corrupted frames in mind. However, most frames need less iteration to converge. After a certain number of iterations, the system gets very little performance improvement with any further iteration. Thus, to reduce the long decoding delay and the decoding power, the stopping criterions, which can timely stop the unnecessary iterations, are put forward [4-9], such as the cross-entropy (CE) stopping criterion [4], the sign-change-ratio (SCR) stopping criterion [5], the hard-decision-aided (HDA) stopping criterion [5], the measurement of reliability (MOR) stopping criterion [6], the convolution-sum (CS) stopping criterion [7], the bitbased partial iteration criterion [8], the Min-CorrEx stopping criterion [9] etc. Although they are proposed initially for turbo receiver, if properly modified, they can also be used for BICM-ID receiver to reduce the unnecessary iterations. Reference [10] has modified the CE stopping criterion successfully to be applied to the BICM-ID receiver. This paper proposes an efficient stopping criterion that based on the absolute value of the difference of log-likelihood-ratio (LLR), which is between the outputs of the $(i)_{t h}$ iteration and the $(i$ -

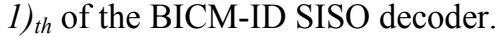

The paper is organized as follows: Section 2 introduces the structure of BICM-ID receiver. Section 3 gives a general review about the widely used CE stopping criterion and section 4 introduces our proposed novel efficient stopping criterion. Section 5 provides the simulation results, which includes the comparisons of our proposed stopping criterion with CE stopping criterion and the fixed scheme. In addition, detailed simulations about various parameters that may affect the decoding performances are also conducted. Finally, section 6 concludes the paper.

\section{The structure of BICM-ID receiver}

The structure of BICM-ID receiver is shown in Figure 1. Before being applied to the SISO decoder, the extrinsic information $L_{e}\left(c_{t}(i)\right)$ of the demapper is deinterleaved. The deinterleaved output is considered as the a priori information $L_{a}\left(c_{t}(i)\right)$ of the SISO decoder, where

$$
\begin{gathered}
L_{a}\left(c_{t}(i)\right)=\log \left[\frac{P\left(c_{t}(i)=0\right)}{P\left(c_{t}(i)=1\right)}\right] \\
L_{e}\left(c_{t}(i)\right)=\log \left[\frac{P\left(c_{t}(i)=0 \mid r_{t}, L_{a}\left(c_{t}\right)\right)}{P\left(c_{t}(i)=1 \mid r_{t}, L_{a}\left(c_{t}\right)\right)}\right]-L_{a}\left(c_{t}(i)\right)
\end{gathered}
$$




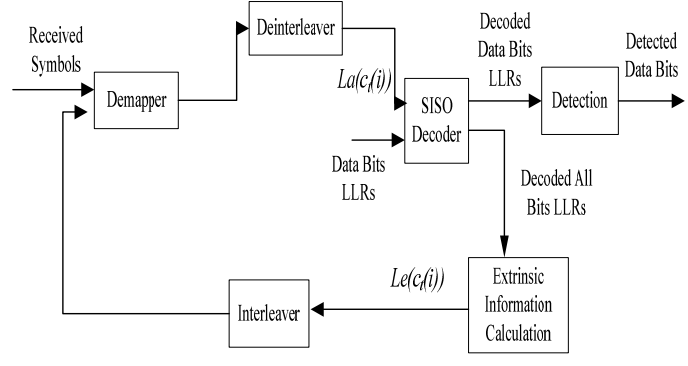

Figure. 1 The structure of BICM-ID receiver

Similarly, the extrinsic information of the SISO decoder is then interleaved and fed back to the demapper as the a prior information of the next iteration.

We note that $L_{a}\left(c_{t}(i)\right)$ is set to be zero during the first iteration as the assumption that the input takes value 0 or 1 with the same probability. At the last iteration, the hard decision of the information bit is ultimately made based on the sign of its LLR value. The iterative process won't stop until the preset maximum iteration number $M$ is reached. This is the conventional fixed iteration number scheme. We call it fixed scheme.

\section{The typical CE stopping criterion}

CE (cross-entropy) is a measurement of how close two distributions are. For the distributions $p$ and $q$ of a finite alphabet $\chi, \mathrm{CE}$ is defined as

$$
H[p, q]=\sum_{x \in \chi} p(x) \log \frac{p(x)}{q(x)}
$$

In BICM-ID, assume that $L_{\mathrm{DEC}}^{i}\left(\hat{c_{k}}\right)$ represents the LLR of the decoded bit $c_{k}(k=1,2,3, \ldots, N)$ after the $i_{t h}$ iteration and $L_{\mathrm{DEC}}^{i-I}\left(\hat{c_{k}}\right)$ represents the LLR of the $(i-1)_{\text {th }}$ iteration respectively. Thus, the CE value between the decoding outputs of the two consecutive iterations is

$$
\begin{gathered}
H\left[P_{D E C}^{i}\left(\hat{c}_{k}^{i}\right), P_{D E C}^{i-1}\left(\hat{c}_{k}^{i-1}\right)\right] \\
=P_{D E C}^{i}\left(\hat{c}_{k}^{i}=0\right) \log \frac{P_{D E C}^{i}\left(\hat{c}_{k}^{i}=0\right)}{P_{D E C}^{i-1}\left(\hat{c}_{k}^{i-1}=0\right)} \\
+P_{D E C}^{i}\left(\hat{c}_{k}^{i}=1\right) \log \frac{P_{D E C}^{i}\left(\hat{c}_{k}^{i}=1\right)}{P_{D E C}^{i-1}\left(\hat{c}_{k}^{i-1}=1\right)}
\end{gathered}
$$

where

$$
P_{D E C}\left(\hat{c}_{k}=0\right)=\frac{e^{L_{D E C}\left(\hat{c}_{k}\right)}}{1+e^{L_{D E C}\left(\hat{c}_{k}\right)}}
$$

$$
P_{D E C}\left(\hat{c}_{k}=1\right)=\frac{1}{1+e^{L_{D E C}\left(\hat{c}_{k}\right)}}
$$

Substituting (5) and (6) into (4), we get CE:

$$
\begin{gathered}
H\left[P_{D E C}^{i}\left(\hat{c}^{i}\right), P_{D E C}^{i-1}\left(\hat{c}^{i-1}\right)\right] \\
=\sum_{k=1}^{N}\left[\frac{L_{D E C}^{i-1}\left(\hat{c}_{k}^{i-1}\right)-L_{D E C}^{i}\left(\hat{c}_{k}^{i}\right)}{1+e^{L_{D E C}^{i}\left(\hat{c}_{k}^{i}\right)}}+\log \frac{1+e^{-L_{D E C}^{i-1}\left(\hat{c}_{k}^{i-1}\right)}}{1+e^{-L_{D E C}^{i}\left(\hat{c}_{k}^{i}\right)}}\right]
\end{gathered}
$$

As the iteration continues, the outputs between the two consecutive iterations $i$ and $i-1$ come to be approximately the same. Therefore, the CE value becomes smaller and smaller. When the iteration proceeds to a certain extent, although the iteration number continues increasing, the CE value won't decrease any more. This means that the iteration has reached the decoding limit. Then, the iterative decoding process can be terminated. We usually set a threshold to effectively stop the unnecessary iterations:

$$
\begin{aligned}
T(i) & =\sum_{k=1}^{N}\left[\frac{L_{D E C}^{i-1}\left(\hat{c}_{k}^{i-1}\right)-L_{D E C}^{i}\left(\hat{c}_{k}^{i}\right)}{1+e^{L_{D E C}^{i}\left(\hat{c}_{k}^{i}\right)}}+\log \frac{1+e^{-L_{D E E}^{i-1}\left(c_{k}^{i-1}\right)}}{1+e^{\left.-L_{D E C}^{i} \hat{c}_{k}^{i}\right)}}\right] \\
& <\text { threshold }
\end{aligned}
$$

\section{The proposed novel efficient stopping criterion}

As introduced in Section 1, the iterative decoding process converges when the iteration proceeds to a certain extent. We suppose that the iterative decoding process converges at iteration $i$, then, we have

$$
\operatorname{sign}\left(L_{D E C}^{i-1}\left(\hat{c}_{k}\right)\right)=\operatorname{sign}\left(L_{D E C}^{i}\left(\hat{c}_{k}\right)\right)
$$

Meanwhile, the difference between the magnitudes of $L_{\mathrm{DEC}}^{i}\left(\hat{c_{k}}\right)$ and $L_{\mathrm{DEC}}^{i-l}\left(\hat{c_{k}}\right)$ is very small, which is less than 1.0. Therefore, the $\triangle L L R(i)$ is small enough to terminate the iterative process, where

$$
\Delta L L R(i)=L_{D E C}^{i-1}\left(\hat{c}_{k}\right)-L_{D E C}^{i}\left(\hat{c_{k}}\right)
$$

We use $|\Delta L L R(i)|$ as the decision element of whether to continue the iterative process or not, where

$$
|\Delta L L R(i)|=\left|L_{D E C}^{i-1}\left(\hat{c}_{k}\right)-L_{D E C}^{i}\left(\hat{c}_{k}\right)\right|
$$


Thus, our proposed stopping criterion can be described as: Compare $|\Delta L L R(i)|$ with a threshold, which is a predetermined sufficiently small value. (In this paper, we set the threshold as $10^{-3}|\Delta L L R(1)|$ ). The iterative cycle is stopped when

$$
|\Delta L L R(i)|<10^{-3}|\Delta L L R(1)|
$$

In the proposed criterion, there is no complex exponential computation in the iterative process, which is much easier for the implementation of the BICM-ID receiver.

\section{Simulation results}

We first introduce the simulation environments: BICM-ID system; rate 1/2 convolutional code; 8 PSK modulation; SP mapping; Rayleigh fading channel; frame size 2048. For stopping criterions, the maximum iteration number $Q$ is set to be 10 . For fixed scheme, the fixed iteration number $M$ is also set to be 10 for comparison. For CE stopping criterion, when $T(i)<10^{-4} T(1)$, stop iteration. For our proposed novel efficient stopping criterion, when $|\Delta L L R(i)|<10^{-3}|\Delta L L R(1)|$, stop iteration.

As shown in Table 1, although the proposed stopping criterion needs about $0.31 \sim 2$ more iteration numbers than $\mathrm{CE}$ criterion, it can save as much as 5 iteration numbers in low and high SNRs (signal-tonoise ratios) compared with the fixed scheme. Meanwhile, Figure 2 shows it can get as excellent performance in terms of BER as CE and the fixed. The superiority existing in our proposed criterion is that without complex exponential computation as $\mathrm{CE}$, it can get coding gains compared with $\mathrm{CE}$ criterion and the fixed at high SNRs as shown clearly in Figure 3. When BER is below $10^{-6}$, the proposed stopping criterion can get nearly $0.15 \mathrm{~dB}$ coding gains than CE stopping criterion. Meanwhile, there is nearly no BER performance degradation between the proposed criterion and the other two schemes at other SNRs. Figure 2 shows that the BER performance curve of our proposed criterion almost coincides with the curves of $\mathrm{CE}$ and the fixed at other SNRs.

Figure $4 \sim 6$ and Table $2 \sim 5$ are a series of simulation results of detailed discussions about the parameters that may affect the decoding performances using proposed stopping criterion.

Table 2 and Figure 4 make a joint display that QPSK modulation performs the best both in terms of BER and average iteration numbers. When BER is at $10^{-4}$, QPSK modulation can get approximately $0.8 \mathrm{~dB}$ and $1.2 \mathrm{~dB}$ coding gains than 8PSK and 16QAM respectively. When SNR is $5.5 \mathrm{~dB}$, QPSK can save about 1.94 and 5.29 iterations than 8PSK and
16QAM. When SNR is $7 \mathrm{~dB}$, the superiority of QPSK is nearly 1.02 and 2.36. At low SNRs, although 16QAM needs less iteration, its BER performance is the worst. However, when SNR is high enough, 16QAM can surpass 8PSK in terms of BER and only require less than 2 iteration numbers for compensation. Consider 8PSK modulation as a representative, besides the reduced iteration numbers, the proposed stopping criterion can get as excellent BER performance as the fixed and even better at high SNRs.

Table 1. Comparison of average iteration numbers

\begin{tabular}{|c|c|c|c|}
\hline & $\begin{array}{c}\text { Fixed } \\
\text { scheme }\end{array}$ & $\begin{array}{c}\text { CE } \\
\text { stopping } \\
\text { criterion }\end{array}$ & $\begin{array}{c}\text { Proposed } \\
\text { stopping } \\
\text { criterion }\end{array}$ \\
\hline $0(\mathrm{~dB})$ & 10 & 4.06 & 5.04 \\
\hline 1 & 10 & 4.87 & 5.97 \\
\hline 2 & 10 & 6.09 & 7.47 \\
\hline 3 & 10 & 9.44 & 9.97 \\
\hline 4 & 10 & 9.65 & 9.96 \\
\hline 5 & 10 & 5.55 & 7.51 \\
\hline 5.5 & 10 & 4.60 & 6.42 \\
\hline 6 & 10 & 4.10 & 5.83 \\
\hline 6.5 & 10 & 3.74 & 5.23 \\
\hline 7 & 10 & 3.29 & 5.02 \\
\hline 7.5 & 10 & 3.09 & 5.00 \\
\hline 8 & 10 & 3.03 & 5.00 \\
\hline 8.5 & 10 & 3.01 & 4.98 \\
\hline 9 & 10 & 3.00 & 4.81 \\
\hline
\end{tabular}

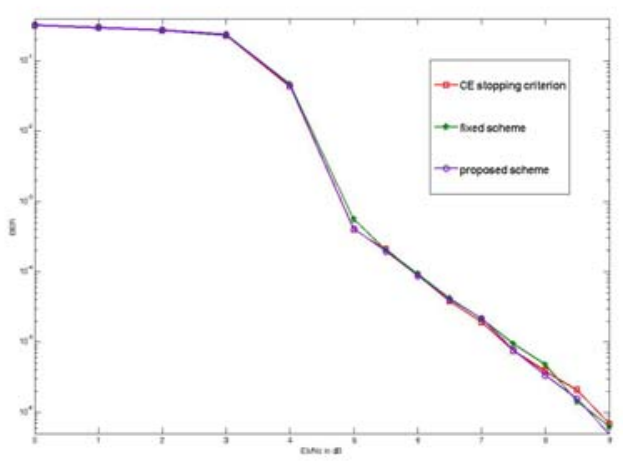

Figure. 2 BER performance comparison

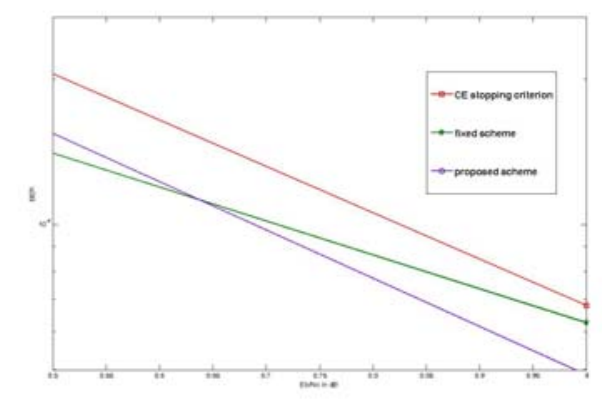

Figure. 3 BER performance comparison at high SNRs 
Table 2. Comparison of average iteration numbers regarding various modulations

\begin{tabular}{|c|c|c|c|c|}
\hline & $\begin{array}{c}\text { Fixed } \\
8 \text { PSK }\end{array}$ & 16 QAM & QPSK & 8 PSK \\
\hline $0(\mathrm{~dB})$ & 10 & 4.17 & 7.16 & 5.04 \\
\hline 1 & 10 & 4.84 & 10 & 5.97 \\
\hline 2 & 10 & 5.11 & 10 & 7.47 \\
\hline 3 & 10 & 6.34 & 8.57 & 9.97 \\
\hline 4 & 10 & 8.94 & 5.74 & 9.96 \\
\hline 5 & 10 & 10 & 4.95 & 7.51 \\
\hline 5.5 & 10 & 9.77 & 4.48 & 6.42 \\
\hline 6 & 10 & 8.50 & 4.10 & 5.83 \\
\hline 6.5 & 10 & 7.21 & 4.01 & 5.23 \\
\hline 7 & 10 & 6.36 & 4.00 & 5.02 \\
\hline 7.5 & 10 & 6.01 & 4.00 & 5.00 \\
\hline 8 & 10 & 5.70 & 4.00 & 5.00 \\
\hline 8.5 & 10 & 5.14 & 4.00 & 4.98 \\
\hline 9 & 10 & 5.01 & & 4.81 \\
\hline
\end{tabular}

Table 3. Comparison of average iteration numbers regarding various mappings

\begin{tabular}{|c|c|c|c|c|c|}
\hline & $\begin{array}{c}\text { Fixed } \\
\text { SP }\end{array}$ & SP & SSP & MSEW & Gray \\
\hline $0(\mathrm{~dB})$ & 10 & 5.04 & 4.00 & 4.00 & 6 \\
\hline 1 & 10 & 5.97 & 4.49 & 4.44 & 7.16 \\
\hline 2 & 10 & 7.47 & 5.09 & 5.06 & 8.99 \\
\hline 3 & 10 & 9.97 & 6.17 & 6.19 & 9.63 \\
\hline 4 & 10 & 9.96 & 8.89 & 8.69 & 8.34 \\
\hline 5 & 10 & 7.51 & 10 & 10 & 6.20 \\
\hline 5.5 & 10 & 6.42 & 9.42 & 9.39 & 5.39 \\
\hline 6 & 10 & 5.83 & 7.84 & 7.85 & 4.92 \\
\hline 6.5 & 10 & 5.23 & 6.84 & 6.85 & 4.53 \\
\hline 7 & 10 & 5.02 & 6.13 & 6.13 & 4.15 \\
\hline 7.5 & 10 & 5.00 & 5.99 & & 4.04 \\
\hline 8 & 10 & 5.00 & & & 4.02 \\
\hline 8.5 & 10 & 4.98 & & & 4.00 \\
\hline 9 & 10 & 4.81 & & & 4.00 \\
\hline
\end{tabular}

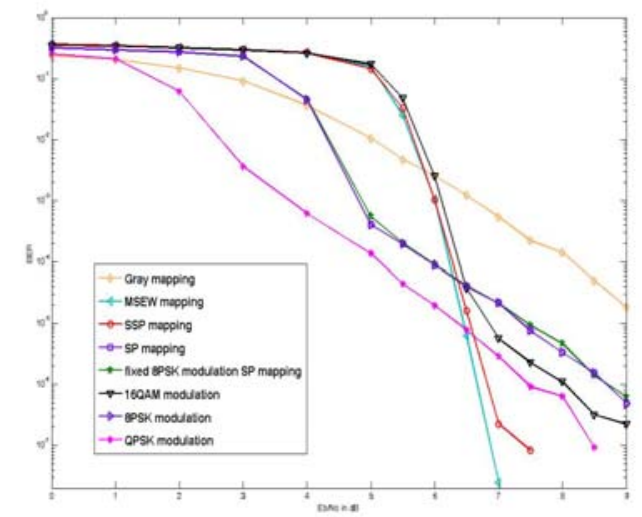

Figure. 4 BER performance comparison regarding various modulations and mappings

It can be seen obviously from Table 3 and Figure 4 that SSP and MSEW mapping schemes can get the best BER performance at high SNR sections, but need more iteration numbers for compensation. SP mapping performs the best in terms of BER at low to medium SNRs. When BER is at $10^{-4}$, compared with SSP and MSEW, SP mapping can get nearly $0.2 \mathrm{~dB}$ coding gains. Meanwhile, it can save iteration numbers at medium SNRs as well. Clearly, Gray mapping isn't suitable for BICM-ID. Take SP mapping as a representative, the scheme that employs proposed stopping criterion not only can greatly reduce the iteration numbers, but also can achieve coding gains at the same time compared with the fixed.

Table 4. Comparison of average iteration numbers regarding various decoding algorithms

\begin{tabular}{|c|c|c|c|c|c|}
\hline & $\begin{array}{c}\text { Fixed } \\
\text { Log- } \\
\text { MAP }\end{array}$ & $\begin{array}{c}\text { Log- } \\
\text { MAP }\end{array}$ & $\begin{array}{c}\text { Linear- } \\
\text { Log- } \\
\text { MAP }\end{array}$ & $\begin{array}{c}\text { Max- } \\
\text { Log- } \\
\text { MAP }\end{array}$ & $\begin{array}{c}\text { Constant- } \\
\text { Log- } \\
\text { MAP }\end{array}$ \\
\hline $0(\mathrm{~dB})$ & 10 & 5.04 & 7.23 & 10 & 10 \\
\hline 1 & 10 & 5.97 & 8.63 & 10 & 10 \\
\hline 2 & 10 & 7.47 & 10 & 10 & 10 \\
\hline 3 & 10 & 9.97 & 10 & 10 & 10 \\
\hline 4 & 10 & 9.96 & 9.92 & 9.96 & 9.94 \\
\hline 5 & 10 & 7.51 & 7.52 & 7.86 & 7.52 \\
\hline 5.5 & 10 & 6.42 & 6.41 & 6.52 & 6.46 \\
\hline 6 & 10 & 5.83 & 5.86 & 5.86 & 5.84 \\
\hline 6.5 & 10 & 5.23 & 5.22 & 5.23 & 5.23 \\
\hline 7 & 10 & 5.02 & 5.01 & 5.02 & 5.02 \\
\hline 7.5 & 10 & 5.00 & 5.00 & 5.00 & 5.00 \\
\hline 8 & 10 & 5.00 & 5.00 & 5.00 & 5.00 \\
\hline 8.5 & 10 & 4.98 & 4.98 & 4.97 & 4.98 \\
\hline 9 & 10 & 4.81 & 4.80 & 4.77 & 4.80 \\
\hline
\end{tabular}

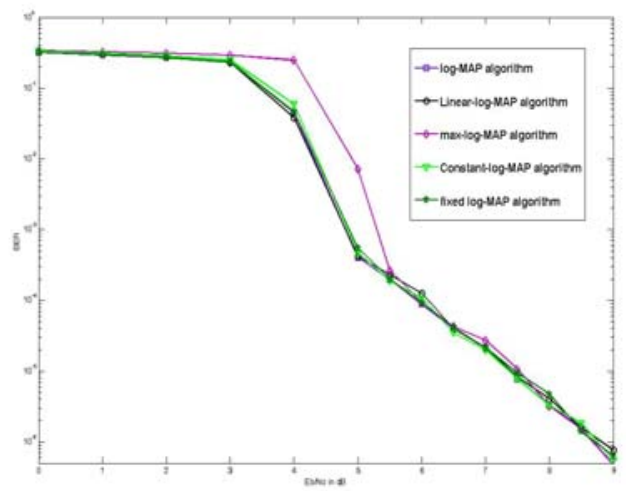

Figure. 5 BER performance comparison regarding various decoding algorithms

As shown in Table 4 and Figure 5, the max-logMAP algorithm performs the worst at medium SNRs both concerning the BER performance and the average iteration numbers. When BER is at $10^{-2}$, other decoding algorithms can achieve more than $0.5 \mathrm{~dB}$ coding gains than max-log-MAP algorithm. At relatively high SNR sections, the four decoding algorithms have almost the same performances considering both BER and iteration numbers. At low SNRs, needing the least iteration numbers, LogMAP algorithm clearly shows its superiority. 
Likewise, with the same decoding algorithm, the proposed criterion has obvious advantages over the fixed both on BER performance and on average iteration numbers.

Table 5. Comparison of average iteration numbers regarding various interleave depths

\begin{tabular}{|c|c|c|c|c|c|c|}
\hline & $\begin{array}{c}\text { Fixed } \\
2048\end{array}$ & 512 & 1024 & 2048 & 4096 & 8192 \\
\hline $0(\mathrm{~dB})$ & 10 & 5.41 & 5.07 & 5.04 & 5.00 & 5.03 \\
\hline 1 & 10 & 6.23 & 6.24 & 5.97 & 5.91 & 5.96 \\
\hline 2 & 10 & 8.53 & 7.84 & 7.47 & 7.47 & 7.33 \\
\hline 3 & 10 & 9.96 & 9.97 & 9.97 & 10 & 10 \\
\hline 4 & 10 & 9.65 & 9.83 & 9.96 & 9.99 & 10 \\
\hline 5 & 10 & 7.57 & 7.42 & 7.51 & 7.60 & 7.58 \\
\hline 5.5 & 10 & 6.45 & 6.42 & 6.42 & 6.40 & 6.40 \\
\hline 6 & 10 & 5.78 & 5.78 & 5.83 & 5.91 & 5.97 \\
\hline 6.5 & 10 & 5.31 & 5.27 & 5.23 & 5.17 & 5.10 \\
\hline 7 & 10 & 5.09 & 5.04 & 5.02 & 5.00 & 5.00 \\
\hline 7.5 & 10 & 5.01 & 5.00 & 5.00 & 5.00 & 5.00 \\
\hline 8 & 10 & 4.95 & 4.99 & 5.00 & 5.00 & 5.00 \\
\hline 8.5 & 10 & 4.82 & 4.92 & 4.98 & 5.00 & 5.00 \\
\hline 9 & 10 & 4.62 & 4.71 & 4.81 & 4.90 & 4.97 \\
\hline
\end{tabular}

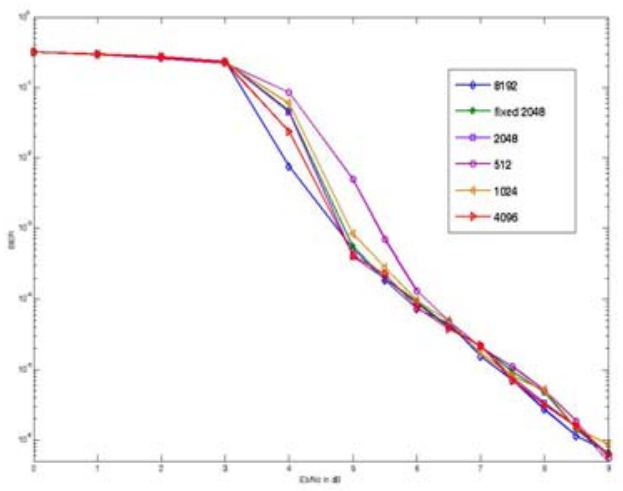

Figure. 6 BER performance comparison regarding various interleave depths

It is revealed clearly from Figure 6 that as the interleave depth increases, the BER performance improves. When BER is at $10^{-2}$, the 1024 case can acquire about $0.35 \mathrm{~dB}$ coding gains compared with the 512 scheme. Likewise, 4096 compares with 2048 and 2048 compares with 1024 both can get nearly $0.1 \mathrm{~dB}$ coding gains. 8192 has an approximately $0.3 \mathrm{~dB}$ superiority compared with 4096 scheme. Table 5 and Figure 6 make a joint show that at relatively high SNR sections, the four interleave curves converge to approximately the same performance bound both concerning BER and average iteration numbers. Similarly, regard the 2048 interleave depth as a representative, the proposed stopping criterion gets superior performances in terms of both BER and average iteration numbers.

\section{Conclusions}

This paper puts forward a novel efficient stopping criterion for BICM-ID system. Detailed discussions concerning various parameters that may affect the decoding performances are also provided. Compared with the fixed scheme, the new simple stopping criterion can not only significantly reduce the iteration numbers but also can get much coding gains in terms of BER at the same time. Although the iteration number is a little more than $\mathrm{CE}$ criterion, it can obtain large coding gains as compensation. Another advantage compared with $\mathrm{CE}$ is that it doesn't need any exponential computation, which can greatly decrease the computation complexity at the receiver. Above all, the excellent performances and the simplicity for receiver implementation make the new proposed criterion more optional in BICMID system.

\section{Acknowledgements}

This paper is supported by the key project of Chinese Ministry of Education (No.106042) and the project sponsored by the Scientific Research Foundation for the Returned Overseas Chinese Scholars, State Education Ministry (2007[24]).

\section{References}

[1] Samahi S.S, Goff S, and Sharif B.S, "Comparative study for bit-interleaved coded modulation with iterative decoding”, IEEE AICT, May 2009, pp. 316-318.

[2] X. Li, J. Ritcey, "Bit-interleaved coded modulation with iterative decoding", Communications Letters, IEEE, vol. 1, no. 6, Nov 1997, pp.169-171.

[3] X. Li and J. Ritcey, "Bit-interleaved coded modulation with iterative decoding using soft feedback," IEE Electronic Letters., vol. 34, no. 10, May 1998, pp.942-943.

[4] J. Hagenauer, E. Offer, and L. Papke, "Iterative decoding of binary block and convolutional codes," IEEE Trans. Inform. Theory, vol. 42, Mar. 1996, pp. 429-445.

[5] R. Y. Shao, S. Lin, and M. P.C. Fossorier, "Two simple stopping criteria for turbo decoding," IEEE Trans. Commun., vol. 47, Aug 1999, pp.1117-1120.

[6] Fan-Min Li, An-Yeu Wu, "A new stopping criterion for efficient early terminationin Turbo decoder designs," ISPACS 2005, Dec 2005, pp.585-588.

[7] Lei Li, Qin Wang, Cheng Youn Lu,“A novel stopping criterion for Turbo decoding," ICICIC 06, First International Conference on, vol. 1, 2006, pp.201-205.

[8] J. Wu, Z. Wang, B. Vojcic,"Partial iterative decoding for binary Turbo codes," Communications, IEEE Trans. Vol.57, No.11, Nov.2009, pp.3298-3306. 
[9] Li Shan, Xie Lei, Chen Huifang, and Wang Kuang, "A new stopping criterion for Duo-binary Turbo codes", CMC. IEEE, Vol.2, 2010, pp.271-274.

[10] S. Zhang, J. Li, C. Cai, "A variable iterative decoding scheme for BICM-ID based on cross-entropy," WCSP 2009. International Conference on, Nov. 2009, pp.1-4. 